\title{
VASCULAR FLORA OF THE OKIĆNICA EDUCATIONAL TRAIL IN ŽUMBERAK-SAMOBORSKO GORJE NATURE PARK (NW CROATIA)
}

\section{Marija Martinović ${ }^{\prime}$, Nikolina Valjak ${ }^{2}$, Vedran Šegota ${ }^{3 *}$, Paolo Bartolić ${ }^{4}$, Elizabeta Jakovac ${ }^{5}$, Petra Stić ${ }^{6}$ \& Katarina Husnjak Malovec ${ }^{7}$}

\author{
${ }^{1}$ Nikole Kramarića 2, 10408 Velika Mlaka, Croatia \\ ${ }^{2}$ Vatrogasna 19, 10380 Sveti Ivan Zelina, Croatia
}

${ }^{3} \mathrm{ZA} \&$ ZAHO herbarium collections, Division of Botany, Department of Biology, Faculty of Science, University of Zagreb, Marulićev trg 20/II, 10000 Zagreb, Croatia

${ }^{4}$ Department of Botany, Charles University, Benátská 2, 12801 Prague, Czech Republic

${ }^{5}$ Gimnazija Gaudeamus - Prva privatna srednja škola u Osijeku, Školska 6, 31000 Zagreb, Croatia

${ }^{6}$ Gruška 22, 10000 Zagreb, Croatia

${ }^{7}$ Public Institution Žumberak-Samoborsko gorje Nature Park, Slani Dol 1, 10430 Samobor, Croatia

Martinović, M., Valjak, N., Šegota, V., Bartolić, P., Jakovac, E., Stić, P. \& Husnjak Malovec, K.: Vascular flora of the educational trail "Okićnica“ in $\mathrm{t}$ he Žumberak-Samoborsko Gorje Nature Park (NW Croatia). Nat. Croat., Vol. 29, No. 1, 109-121, Zagreb, 2020.

Vascular flora of the $2.5 \mathrm{~km}$ long circular Okićnica educational trail in Žumberak-Samoborsko gorje Nature Park contains 287 species and subspecies, with Fabaceae, Asteraceae and Rosaceae the most abundant families. The domination of Eurasiatic and Central European floral elements reflects the phyto-geographical position of the area; however, the presence of Illyricoid plants of mesophylous forests on the NW border of Dinarides is what makes the flora unique. The most common life forms (hemicryptophytes, phanerophytes and geophytes) are the result of a moderate climate and the dominant forest vegetation. Six vulnerable, five near threatened, ten strictly protected, two endemic and nine invasive taxa were recorded along the trail. Our results contribute to the knowledge on the flora of the Nature Park and can be used in the future design of an educational botanical trail.

Key words: biodiversity, Illyrian chorotype, NW Dinarides, Okić

Martinović, M., Valjak, N., Šegota, V., Bartolić, P., Jakovac, E., Stić, P. \& Husnjak Malovec, K.: Vaskularna flora edukativne staze "Okićnica“ u Parku prirode Žumberak-Samoborsko Gorje (SZ Hrvatska). Nat. Croat., Vol. 29, No. 1, 109-121, Zagreb, 2020.

Vaskularna flora 2,5 km duge edukativne kružne staze "Okićnica” u Parku prirode Žumberak-Samoborsko gorje broji 287 vrsta i podvrsta, a najbrojnije porodice su Fabaceae, Asteraceae i Rosaceae. Dominacija euroazijskog i srednjoeuropskog flornog elementa odražava fitogeografski položaj istraživanog područja, no jedinstvenost flore čine ilirikoidne vrste mezofilnih šuma na SZ granici Dinarida. Najčešći životni oblici su hemikriptofiti, fanerofiti i geofiti koji ukazuju na umjerenu klimu i dominaciju šumske vegetacije. Uzduž istraživane staze zabilježeno je šest osjetljivih, pet gotovo ugroženih, deset strogo zaštićenih, dvije endemične i devet invazivnih svojti. Rezultati ovog istraživanja prilog su poznavanju flore Parka prirode i mogu biti temelj za planiranje buduće edukativne botaničke staze.

Ključne riječi: bioraznolikost, ilirski florni element, SZ Dinaridi, Okić

\footnotetext{
* corresponding author: vedran.segota@biol.pmf.hr
} 


\section{INTRODUCTION}

Žumberak-Samoborsko gorje Nature Park is situated in north-western Croatia and encompasses both the southern parts of Žumberačko gorje Mts (Mt Žumberak), as well as Samoborsko Gorje Mts, across its total area of $333 \mathrm{~km}^{2}$. Both mountains are part of Žumberačka Gora (BUZJAK, 2011), a 40 km long massif stretching between the rivers Sava and Kupa in Croatia, and the River Krka in Slovenia. The elevation of the mountain ranges from $180 \mathrm{~m}$ up to $1,178 \mathrm{~m}$ (Sveta Gera peak). It is oriented in the NE-SW direction, and is characterized by Dinaric tectonics and karst formations (VRBEK, 2005). The climate of the area is moderately warm and rainy, without extremely dry periods. The average annual precipitation ranges between 1,100 and 1,700 mm (Penzar \& PenZAR, 1982). The area belongs to the colline and montane forest belt (HORVAT, 1962; ŠUGAR, 1972; VUKELIĆ, 2012).

Phytogeographically, Mt Žumberačka gora is a bridge between the SE Alps and the NW Dinarides, with many alpine elements on low altitudes and Dinaric elements on the NW border of their distribution (Horvat, 1929; Trinajstić, 1995). The largest part of the area is covered by forests, dominated by sessile oak (Quercus petraea (Matt.) Liebl.) on lower and beech (Fagus sylvatica L.) on higher elevations. Most of the grassland areas are not of natural origin but were created as a result of long lasting deforestation during the centuries. Recently, rapid succession towards shrub and forest vegetation has been taking place, due to the abandonment of traditional land use (pasturing and mowing). The flora of Samoborsko Gorje Mts has previously been investigated by ŠUGAR (1972) (890 taxa), while systematic research of Mt Žumberačka Gora revealed as many as 1,006 plant taxa (VRBEK, 2005). Several recent field studies have focused on the flora of various specific habitats, e.g. pits and cave entrances (BuzJAK et al., 2010), collapsed dolines (BuzJaK et al., 2011), fens (ŠošTarić et al., 2012), springs and ponds (ŠošTARIĆ et al., 2011), waterfalls and rivulets (ŠošTARIĆ et al., 2013) or the flora of certain plant groups, e.g. orchids (VRbeK \& Fiedler, 1998) and bryophytes (Alegro et al., 2015). In 1999, Nature Park of Žumberačko gorje Mts, with the adjacent Samoborsko gorje Mts, was established.

The aim of this research was to study and analyse the flora along the 2.5 long Okićnica Educational Trail, which is situated around the hill of Okić (halfway between the towns of Samobor and Jastrebarsko). The trail is beneath the old medieval castle Stari grad Okić located on the peak of this hill at $495 \mathrm{~m}$ a. s. 1., representing a valuable example of Romanesque architecture in continental Croatia. The circular Okićnica Educational Trail was opened in spring 2017, presenting the geology, history, archaeology and ethnology of the Okić region in ten educational panels (Fig 1.). The collected data are the basis for the future design of an educational botanical trail.

\section{MATERIAL AND METHODS}

The field study was conducted from April to June 2018, within a student field project undertaken as a part of the practical course Flora of Croatia that forms part of the undergraduate curriculum of the Faculty of Science in Zagreb.

Most of the plant material was identified in the field, although several specimens were collected and dried for further precise identification in the lab, while strictly protected specimens (e.g. orchids) were photographed only. Identification was performed using standard literature (Horvatić, 1954; Delforge, 1995; Domac, 1994; JAVOR- 
KA \& Csapody, 1991; Rothmaler, 2000; Eggenberg \& MöHL, 2007). Nomenclature follows the Flora Croatica Database (Nikolić, 2020). Authors of all the taxa names are given in the species list in Tab. 1, but not within the text. All collected plant material was deposited in Herbarium Croaticum collection (ZA) and is publicly available via the Flora Croatica Database and Virtual Herbarium (REšETNIK \& ŠEGOTA, 2020).

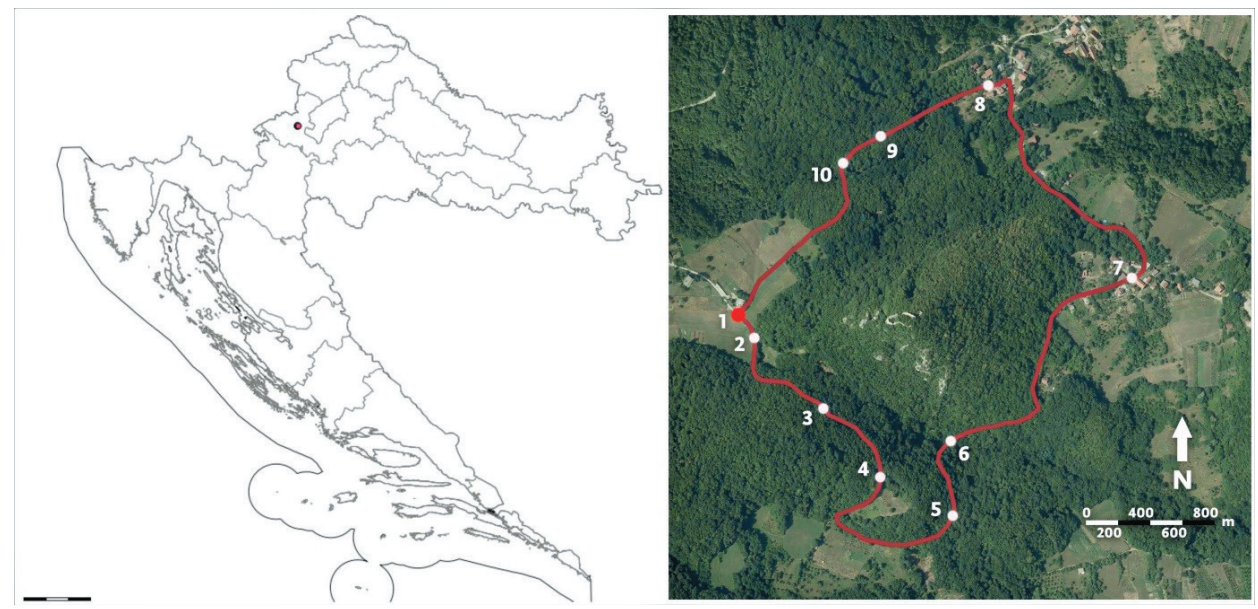

Fig. 1. Geographical position of the studied area in Croatia (left) and the shape of the Okićnica trail (right).

Chorology is based on Horvatić (1963) and Horvatić et al. (1967-1968), with some recent revisions following LANDOLT et al. (2010). Life forms, as defined by RAUNKIAER (1934) (phanerophytes, chamaephytes, hemicryptophytes, therophytes and geophytes) were taken from the Flora Croatica Database (Nikolić, 2020), based on Ellenberg \& LAUSCHNER (2010). Several updates were made according to the recent approaches of Alegro et al. (2013) and Vuković et al. (2013). Endemic taxa were checked according to Nikolić et al. (2015) and Nikolić (2020), invasive taxa according to Nikolić et al. (2014) and Nikolić (2020), legally protected taxa according to Anonymous $(2013,2016)$ and threatened taxa according to Nikolić \& Topić (2005).

\section{RESULTS AND DISCUSSION}

In the course of our research, 287 plant taxa of vascular plants from 74 families were recorded along the circular "Okićnica Educational Trail" (Tab. 1). The trail is placed mostly within floristically rich beech-dominated forests; however, several segments going through dry grasslands and along traditional vineyards and orchards as well as the pronounced anthropogenic impact on the trail contribute considerably to species richness. Since the whole area was not investigated evenly, only the flora along the trail, comparisons with the flora of other similar areas were not applicable.

The vast majority of taxa (98\%) belong to Spermatophyta (Pinidae and Magnoliidae), while only eight belong to Pterydophyta (Polypodiidae and Equisetidae). Most taxa are members of Fabaceae (8.04\%), Asteraceae (7.34\%), Rosaceae (6.99\%), Lamiaceae and Poaceae (5.94\% each) (Fig. 2). 
Tab. 1. List of vascular flora of the trail "Okićnica". Chorotypes (1 - Mediterranean, 2 - Illyrian-Balkan, 3 - South European, 4 - Atlantic, 5 - East European-Pontic, 6 - Southeastern European, 7 - Central European, 8 - European, 9 - Eurasiatic, 10 - circumholoarctic, 11 - cosmopolits, 12 - adventive and cultivated taxa), life forms ( $\mathrm{P}$ - phanerophytes, $\mathrm{Ch}$ - chamaephytes, $\mathrm{H}$ - hemicryptophytes, $\mathrm{G}$ - geophytes, T - therophytes), IUCN category (LC - least concern, NT - near threatened, VU - vulnerable), legal protection (SP - strictly protected), endemism (E), invasive taxa (I).

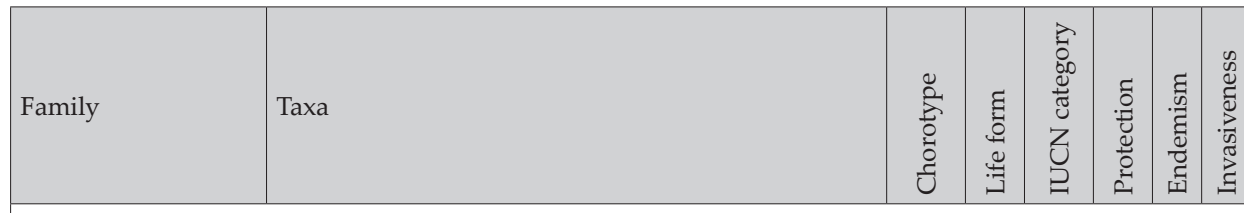

PTERIDOPHYTA

\begin{tabular}{|c|c|c|c|c|c|c|}
\hline \multirow{2}{*}{ Aspleniaceae } & Asplenium scolopendrium L. & 10 & $\mathrm{H}$ & & & \\
\hline & Asplenium trichomanes $\mathrm{L}$. & 11 & $\mathrm{H}$ & & & \\
\hline Dryopteridaceae & Polystichum aculeatum (L.) Roth & 11 & $\mathrm{H}$ & & & \\
\hline \multirow{2}{*}{ Equisetaceae } & Equisetum arvense $\mathrm{L}$. & 10 & G & & & \\
\hline & Equisetum telmateia Ehrh. & 10 & G & & & \\
\hline Hypolepidaceae & Pteridium aquilinum (L.) Kuhn & 11 & G & & & \\
\hline Polypodiaceae & Polypodium vulgare $\mathrm{L}$. & 11 & $\mathrm{H}$ & & & \\
\hline Woodsiaceae & Athyrium filix-femina (L.) Roth & 9 & $\mathrm{H}$ & & & \\
\hline \multicolumn{7}{|c|}{ SPERMATOPHYTA } \\
\hline \multirow{3}{*}{ Aceraceae } & Acer campestre $\mathrm{L}$. & 9 & $\mathrm{P}$ & & & \\
\hline & Acer obtusatum Willd. & 2 & $\mathrm{P}$ & & & \\
\hline & Acer pseudoplatanus L. & 8 & $\mathrm{P}$ & & & \\
\hline \multirow{2}{*}{ Amaryllidaceae } & Allium ursinum $\mathrm{L}$. & 7 & G & & & \\
\hline & Galanthus nivalis L. & 9 & G & LC & & \\
\hline \multirow{6}{*}{ Apiaceae } & Aegopodium podagraria $\mathrm{L}$. & 9 & $\mathrm{H}$ & & & \\
\hline & Anthriscus sylvestris (L.) Hoffm. & 7 & $\mathrm{H}$ & & & \\
\hline & Daucus carota $\mathrm{L}$. & 1 & $\mathrm{H}$ & & & \\
\hline & Hacquetia epipactis (Scop.) DC. & 2 & $\mathrm{H}$ & & & \\
\hline & Heracleum sphondylium L. & 9 & $\mathrm{H}$ & & & \\
\hline & Sanicula europaea L. & 9 & $\mathrm{H}$ & & & \\
\hline \multirow{2}{*}{ Apocynaceae } & Vinca major $\mathrm{L}$. & 12 & $\mathrm{Ch}$ & & & \\
\hline & Vinca minor $\mathrm{L}$. & 7 & $\mathrm{Ch}$ & & & \\
\hline Aquifoliaceae & Ilex aquifolium $\mathrm{L}$. & 3 & $\mathrm{P}$ & VU & SP & \\
\hline Araceae & Arum maculatum L. & 7 & G & & & \\
\hline Araliaceae & Hedera helix L. & 7 & $\mathrm{P}$ & & & \\
\hline Aristolochiaceae & Asarum europaeum L. & 9 & $\mathrm{H}$ & & & \\
\hline \multirow{5}{*}{ Asparagaceae } & Ornithogalum pyramidale $\mathrm{L}$. & 3 & G & & & \\
\hline & Ornithogalum umbellatum $\mathrm{L}$. & 3 & G & & & \\
\hline & Polygonatum multiflorum (L.) All. & 9 & G & & & \\
\hline & Ruscus hypoglossum L. & 12 & G & NT & & \\
\hline & Scilla bifolia $\mathrm{L}$. & 3 & G & & & \\
\hline \multirow{8}{*}{ Asteraceae } & Achillea millefolium $\mathrm{L}$. & 9 & $\mathrm{H}$ & & & \\
\hline & Ambrosia artemisiifolia L. & 12 & $\mathrm{~T}$ & & & I \\
\hline & Arctium lappa $\mathrm{L}$. & 9 & $\mathrm{H}$ & & & \\
\hline & Artemisia verlotiorum Lamotte & 12 & $\mathrm{H}$ & & & I \\
\hline & Artemisia vulgaris L. & 9 & $\mathrm{H}$ & & & \\
\hline & Bellis perennis L. & 9 & $\mathrm{H}$ & & & \\
\hline & Buphthalmum salicifolium L. & 7 & $\mathrm{H}$ & & & \\
\hline & Centaurea jacea L. & 9 & $\mathrm{H}$ & & & \\
\hline
\end{tabular}




\begin{tabular}{|c|c|c|c|c|c|c|c|}
\hline Family & Taxa & 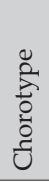 & 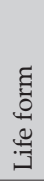 & $\begin{array}{l}3 \\
0 \\
8 \\
0 \\
\Xi \\
Z \\
Z \\
己 \\
己\end{array}$ & 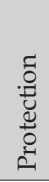 & 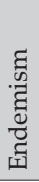 & 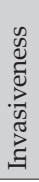 \\
\hline \multirow{13}{*}{ Asteraceae } & Chamomilla suaveolens (Pursh) Rydb. & 12 & $\mathrm{~T}$ & & & & $\mathrm{I}$ \\
\hline & Cirsium arvense (L.) Scop. & 9 & G & & & & \\
\hline & Cirsium vulgare (Savi) Ten. & 9 & $\mathrm{H}$ & & & & \\
\hline & Conyza canadensis (L.) Cronquist & 9 & $\mathrm{H}$ & & & & $\mathrm{I}$ \\
\hline & Doronicum austriacum Jacq. & 3 & $\mathrm{H}$ & & & & \\
\hline & Erigeron annuus (L.) Pers. & 12 & $\mathrm{H}$ & & & & $\mathrm{I}$ \\
\hline & Leucanthemum vulgare Lam. & 9 & $\mathrm{H}$ & & & & \\
\hline & Petasites albus (L.) Gaertn. & 7 & G & & & & \\
\hline & Petasites hybridus (L.) P. Gaertn. , B. Mey. et Schreb. & 9 & G & & & & \\
\hline & Senecio ovatus (P. Gaertn., B. Mey. et Scherb.) Willd. & 7 & $\mathrm{H}$ & & & & \\
\hline & Solidago gigantea Aiton & 12 & $\mathrm{H}$ & & & & $\mathrm{I}$ \\
\hline & Tanacetum corymbosum (L.) Sch. Bip. & 7 & $\mathrm{H}$ & & & & \\
\hline & Tussilago farfara $\mathrm{L}$. & 9 & $\mathrm{H}$ & & & & \\
\hline \multirow{2}{*}{ Betulaceae } & Alnus glutinosa (L.) Gaertn. & 9 & $\mathrm{P}$ & & & & \\
\hline & Betula pendula Roth & 9 & $\mathrm{P}$ & & & & \\
\hline \multirow{5}{*}{ Boraginaceae } & Lithospermum purpurocaeruleum L. & 3 & $\mathrm{H}$ & & & & \\
\hline & Myosotis sparsiflora Pohl & 9 & $\mathrm{~T}$ & & & & \\
\hline & Pulmonaria officinalis L. & 6 & $\mathrm{H}$ & & & & \\
\hline & Symphytum officinale L. & 8 & $\mathrm{H}$ & & & & \\
\hline & Symphytum tuberosum L. & 3 & G & & & & \\
\hline \multirow{11}{*}{ Brassicaceae } & Alliaria petiolata (M. Bieb.) Cavara et Grande & 9 & $\mathrm{H}$ & & & & \\
\hline & Armoracia rusticana P. Gaertn. , B. Mey. et Scherb. & 11 & G & & & & \\
\hline & Capsella bursa-pastoris (L.) Medik. & 11 & $\mathrm{~T}$ & & & & \\
\hline & Cardamine bulbifera (L.) Crantz & 7 & G & & & & \\
\hline & Cardamine enneaphyllos (L.) Crantz & 3 & G & & & & \\
\hline & Cardamine hirsuta L. & 1 & $\mathrm{~T}$ & & & & \\
\hline & Cardamine impatiens L. & 9 & $\mathrm{H}$ & & & & \\
\hline & Cardamine trifolia $\mathrm{L}$. & 2 & G & & & & \\
\hline & Lepidium virginicum $\mathrm{L}$. & 12 & $\mathrm{~T}$ & & & & $\mathrm{I}$ \\
\hline & Rorippa sylvestris (L.) Besser & 9 & $\mathrm{H}$ & & & & \\
\hline & Thlaspi alliaceum $\mathrm{L}$. & 3 & $\mathrm{~T}$ & & & & \\
\hline \multirow{4}{*}{ Campanulaceae } & Campanula patula L. & 7 & $\mathrm{H}$ & & & & \\
\hline & Campanula rapunculus L. & 9 & $\mathrm{H}$ & & & & \\
\hline & Campanula trachelium L. & 9 & $\mathrm{H}$ & & & & \\
\hline & Phyteuma spicatum L. & 7 & $\mathrm{H}$ & & & & \\
\hline \multirow{5}{*}{ Cannabaceae } & Humulus lupulus L. & 9 & $\mathrm{H}$ & & & & \\
\hline & Lonicera caprifolium L. & 3 & $\mathrm{P}$ & & & & \\
\hline & Sambucus ebulus L. & 1 & $\mathrm{H}$ & & & & \\
\hline & Sambucus nigra L. & 7 & $\mathrm{P}$ & & & & \\
\hline & Viburnum lantana L. & 3 & $\mathrm{P}$ & & & & \\
\hline \multirow{6}{*}{ Caryophyllaceae } & Arenaria serpyllifolia $\mathrm{L}$. & 11 & $\mathrm{~T}$ & & & & \\
\hline & Cerastium glomeratum Thuill. & 1 & $\mathrm{~T}$ & & & & \\
\hline & $\begin{array}{l}\text { Dianthus giganteus D' Urv ssp. croaticus (Borbás) } \\
\text { Tutin }\end{array}$ & 6 & $\mathrm{H}$ & VU & SP & $\mathrm{E}$ & \\
\hline & Lychnis flos-cuculi L. & 9 & $\mathrm{H}$ & & & & \\
\hline & Moenchia mantica (L.) Bartl. & 3 & $\mathrm{~T}$ & & & & \\
\hline & Myosoton aquaticum (L.) Moench & 9 & $\mathrm{H}$ & & & & \\
\hline
\end{tabular}




\begin{tabular}{|c|c|c|c|c|c|c|c|}
\hline Family & Taxa & 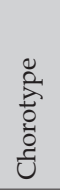 & 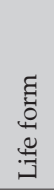 & 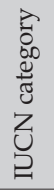 & 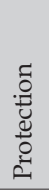 & 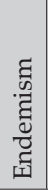 & 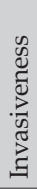 \\
\hline \multirow{3}{*}{ Caryophyllaceae } & Silene italica (L.) Pers. & 6 & $\mathrm{H}$ & & & & \\
\hline & Stellaria holostea $\mathrm{L}$. & 9 & $\mathrm{Ch}$ & & & & \\
\hline & Stellaria media (L.) Vill. & 1 & $\mathrm{~T}$ & & & & \\
\hline Celastraceae & Euonymus europaeus L. & 9 & $\mathrm{P}$ & & & & \\
\hline Chenopodiaceae & Chenopodium album $\mathrm{L}$. & 11 & $\mathrm{~T}$ & & & & \\
\hline \multirow{12}{*}{ Cichoriaceae } & Aposeris foetida (L.) Less. & 3 & $\mathrm{H}$ & & & & \\
\hline & Crepis biennis L. & 7 & $\mathrm{H}$ & & & & \\
\hline & Hieracium murorum L. & 9 & $\mathrm{H}$ & & & & \\
\hline & Hieracium pilosella $\mathrm{L}$. & 5 & G & & & & \\
\hline & Hieracium praealtum Vill. ex Gochnat & 9 & $\mathrm{H}$ & & & & \\
\hline & Lactuca serriola L. & 11 & $\mathrm{H}$ & & & & \\
\hline & Lapsana communis L. & 9 & $\mathrm{H}$ & & & & \\
\hline & Mycelis muralis (L.) Dumort. & 7 & $\mathrm{H}$ & & & & \\
\hline & Prenanthes purpurea $\mathrm{L}$. & 7 & $\mathrm{H}$ & & & & \\
\hline & Sonchus oleraceus L. & 9 & $\mathrm{~T}$ & & & & \\
\hline & Taraxacum officinale Weber & 9 & $\mathrm{H}$ & & & & \\
\hline & Tragopogon pratensis L. ssp. orientalis (L.) Čelak. & 9 & $\mathrm{H}$ & & & & \\
\hline Cistaceae & $\begin{array}{l}\text { Helianthemum nummularium (L.) Mill. ssp. obscurum } \\
\text { (Čelak.) Holub }\end{array}$ & 3 & $\mathrm{Ch}$ & & & & \\
\hline \multirow{2}{*}{ Clusiaceae } & Hypericum hirsutum $\mathrm{L}$. & 11 & $\mathrm{H}$ & & & & \\
\hline & Hypericum perforatum $\mathrm{L}$. & 9 & $\mathrm{H}$ & & & & \\
\hline Colchicaceae & Colchicum autumnale $\mathrm{L}$. & 7 & G & & & & \\
\hline Convolvulaceae & Convolvulus arvensis $\mathrm{L}$. & 11 & $\mathrm{H}$ & & & & \\
\hline \multirow{2}{*}{ Cornaceae } & Cornus mas L. & 3 & $\mathrm{P}$ & & & & \\
\hline & Cornus sanguinea $\mathrm{L}$. & 8 & $\mathrm{P}$ & & & & \\
\hline \multirow{2}{*}{ Corylaceae } & Carpinus betulus L. & 7 & $\mathrm{P}$ & & & & \\
\hline & Corylus avellana $\mathrm{L}$. & 8 & $\mathrm{P}$ & & & & \\
\hline \multirow{2}{*}{ Crassulaceae } & Sedum acre L. & 11 & $\mathrm{Ch}$ & & & & \\
\hline & Sedum sexangulare $\mathrm{L}$. & 7 & $\mathrm{Ch}$ & & & & \\
\hline Cupressaceae & Juniperus communis L. & 10 & $\mathrm{P}$ & & & & \\
\hline \multirow{8}{*}{ Cyperaceae } & Carex digitata $\mathrm{L}$. & 9 & $\mathrm{H}$ & & & & \\
\hline & Carex flacca Schreb. ssp. flacca & 11 & G & & & & \\
\hline & Carex hallerana Asso & 3 & $\mathrm{H}$ & & & & \\
\hline & Carex hirta L. & 7 & $\mathrm{H}$ & & & & \\
\hline & Carex muricata $\mathrm{L}$. & 9 & $\mathrm{H}$ & & & & \\
\hline & Carex otrubae Podp. & 6 & $\mathrm{H}$ & & & & \\
\hline & Carex spicata Huds. & 9 & $\mathrm{H}$ & & & & \\
\hline & Carex sylvatica Huds. & 9 & $\mathrm{H}$ & & & & \\
\hline Dioscoreaceae & Tamus communis L. & 3 & G & & & & \\
\hline Dipsacaceae & Knautia drymeia Heuff. & 2 & $\mathrm{H}$ & & & & \\
\hline \multirow{6}{*}{ Euphorbiaceae } & Euphorbia amygdaloides L. & 7 & $\mathrm{Ch}$ & & & & \\
\hline & Euphorbia cyparissias L. & 9 & $\mathrm{H}$ & & & & \\
\hline & Euphorbia dulcis L. & 5 & $\mathrm{H}$ & & & & \\
\hline & Euphorbia verrucosa L. & 8 & $\mathrm{H}$ & & & & \\
\hline & Euphorbia virgata Waldst. et Kit. & 9 & $\mathrm{H}$ & & & & \\
\hline & Mercurialis perennis $\mathrm{L}$. & 8 & G & & & & \\
\hline
\end{tabular}




\begin{tabular}{|c|c|c|c|c|c|c|c|}
\hline Family & Taxa & 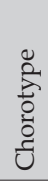 & 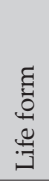 & 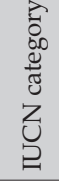 & 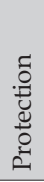 & 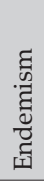 & 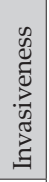 \\
\hline \multirow{23}{*}{ Fabaceae } & Astragalus glycyphyllos L. & 9 & $\mathrm{H}$ & & & & \\
\hline & Chamaecytisus hirsutus (L.) Link & 9 & $\mathrm{Ch}$ & & & & \\
\hline & Coronilla varia $\mathrm{L}$. & 8 & $\mathrm{H}$ & & & & \\
\hline & Dorycnium germanicum (Gremli) Rikli & 3 & $\mathrm{H}$ & & & & \\
\hline & Genista tinctoria L. & 9 & G & & & & \\
\hline & Lathyrus niger (L.) Bernhardt & 8 & G & & & & \\
\hline & Lathyrus pratensis L. & 9 & $\mathrm{H}$ & & & & \\
\hline & Lathyrus vernus (L.) Bernhardt & 1 & $\mathrm{H}$ & & & & \\
\hline & Lembotropis nigricans (L.) Griseb. & 5 & $\mathrm{P}$ & & & & \\
\hline & Lotus corniculatus L. & 11 & $\mathrm{H}$ & & & & \\
\hline & Medicago falcata $\mathrm{L}$. & 9 & $\mathrm{H}$ & & & & \\
\hline & Medicago lupulina L. & 1 & $\mathrm{~T}$ & & & & \\
\hline & Medicago sativa L. & 11 & $\mathrm{H}$ & & & & \\
\hline & Robinia pseudoacacia L. & 12 & $\mathrm{P}$ & & & & $\mathrm{I}$ \\
\hline & Trifolium campestre Schreber & 11 & $\mathrm{~T}$ & & & & \\
\hline & Trifolium montanum L. & 9 & $\mathrm{H}$ & & & & \\
\hline & Trifolium pratense $\mathrm{L}$. & 9 & $\mathrm{H}$ & & & & \\
\hline & Trifolium repens L. & 9 & $\mathrm{H}$ & & & & \\
\hline & Trifolium rubens L. & 9 & $\mathrm{H}$ & & & & \\
\hline & Vicia dumetorum L. & 9 & $\mathrm{H}$ & & & & \\
\hline & Vicia oroboides Wulfen & 2 & $\mathrm{H}$ & & & & \\
\hline & Vicia sativa $\mathrm{L}$. & 1 & $\mathrm{~T}$ & & & & \\
\hline & Vicia villosa Roth ssp. varia (Host) Corb. & 1 & $\mathrm{~T}$ & & & & \\
\hline \multirow{4}{*}{ Fagaceae } & Castanea sativa Mill. & 3 & $\mathrm{P}$ & & & & \\
\hline & Fagus sylvatica L. & 7 & $\mathrm{P}$ & & & & \\
\hline & Quercus cerris L. & 7 & $\mathrm{P}$ & & & & \\
\hline & Quercus petraea (Matt.) Liebl. & 7 & $\mathrm{P}$ & & & & \\
\hline Fumariaceae & Corydalis bulbosa (L.) DC. & 9 & G & & & & \\
\hline Gentianaceae & Gentiana asclepiadea L. & 3 & $\mathrm{H}$ & NT & & & \\
\hline \multirow{4}{*}{ Geraniaceae } & Geranium columbinum L. & 9 & $\mathrm{~T}$ & & & & \\
\hline & Geranium phaeum L. & 3 & $\mathrm{H}$ & & & & \\
\hline & Geranium pusillum Burm. f. & 9 & $\mathrm{~T}$ & & & & \\
\hline & Geranium robertianum $\mathrm{L}$. & 10 & $\mathrm{H}$ & & & & \\
\hline Juglandaceae & Juglans regia L. & 12 & $\mathrm{P}$ & & & & \\
\hline \multirow{3}{*}{ Juncaceae } & Juncus inflexus L. & 9 & $\mathrm{H}$ & & & & \\
\hline & Luzula campestris (L.) DC. & 9 & $\mathrm{H}$ & & & & \\
\hline & Luzula luzuloides (Lam.) Dandy et Wilmott & 7 & $\mathrm{H}$ & & & & \\
\hline \multirow{9}{*}{ Lamiaceae } & Ajuga reptans L. & 9 & $\mathrm{H}$ & & & & \\
\hline & Betonica officinalis L. ssp. serotina (Host) Murb. & 2 & $\mathrm{H}$ & & & & \\
\hline & Clinopodium vulgare $\mathrm{L}$. & 11 & $\mathrm{H}$ & & & & \\
\hline & Glechoma hederacea L. & 9 & $\mathrm{H}$ & & & & \\
\hline & Glechoma hirsuta Waldst. et Kit. & 3 & $\mathrm{H}$ & & & & \\
\hline & Lamium galeobdolon (L.) L. & 5 & $\mathrm{H}$ & & & & \\
\hline & Lamium maculatum L. & 9 & $\mathrm{H}$ & & & & \\
\hline & Lamium orvala $\mathrm{L}$. & 2 & $\mathrm{H}$ & & & & \\
\hline & Lamium purpureum L. & 1 & $\mathrm{~T}$ & & & & \\
\hline
\end{tabular}




\begin{tabular}{|c|c|c|c|c|c|c|c|}
\hline Family & Taxa & 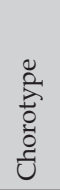 & 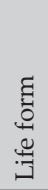 & $\begin{array}{l}\vec{D} \\
0 \\
0 \\
\tilde{J} \\
Z \\
己 \\
己\end{array}$ & 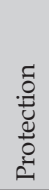 & 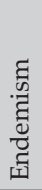 & 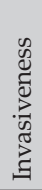 \\
\hline \multirow{8}{*}{ Lamiaceae } & Melittis melissophyllum L. & 7 & $\mathrm{H}$ & & & & \\
\hline & Mentha arvensis L. & 10 & $\mathrm{H}$ & & & & \\
\hline & Prunella vulgaris $\mathrm{L}$. & 10 & $\mathrm{H}$ & & & & \\
\hline & Salvia glutinosa $\mathrm{L}$. & 9 & $\mathrm{H}$ & & & & \\
\hline & Salvia pratensis $\mathrm{L}$. & 8 & $\mathrm{H}$ & & & & \\
\hline & Stachys sylvatica $\mathrm{L}$. & 9 & $\mathrm{H}$ & & & & \\
\hline & Teucrium chamaedrys $\mathrm{L}$. & 3 & $\mathrm{Ch}$ & & & & \\
\hline & Thymus pulegioides L. & 3 & $\mathrm{Ch}$ & & & & \\
\hline \multirow{2}{*}{ Liliaceae } & Gagea lutea (L.) Ker Gawl. & 9 & G & & & & \\
\hline & Lilium martagon $\mathrm{L}$. & 9 & G & VU & SP & & \\
\hline Linaceae & Linum catharticum $\mathrm{L}$. & 11 & $\mathrm{~T}$ & & & & \\
\hline Loranthaceae & Loranthus europaeus Jacq. & 9 & $\mathrm{P}$ & & & & \\
\hline Malvaceae & Hibiscus syriacus $\mathrm{L}$. & 12 & $P$ & & & & \\
\hline Melanthiaceae & Paris quadrifolia $\mathrm{L}$. & 9 & G & & & & \\
\hline \multirow{2}{*}{ Moraceae } & Ficus carica L. & 1 & $\mathrm{P}$ & & & & \\
\hline & Morus alba $\mathrm{L}$. & 12 & $\mathrm{P}$ & & & & \\
\hline \multirow{3}{*}{ Oleaceae } & Forsythia europaea Degen et Bald. & 12 & $\mathrm{P}$ & LC & & & \\
\hline & Fraxinus ornus L. & 3 & $P$ & & & & \\
\hline & Ligustrum vulgare L. & 7 & $\mathrm{P}$ & & & & \\
\hline Onagraceae & Circaea lutetiana $\mathrm{L}$. & 11 & $\mathrm{H}$ & & & & \\
\hline \multirow{6}{*}{ Orchidaceae } & Himantoglossum adriaticum H. Baumann & 6 & G & NT & SP & & \\
\hline & Neottia nidus-avis (L.) Rich. & 9 & G & & SP & & \\
\hline & Orchis morio L. ssp. picta (Loisel.) K. Richt. & 7 & G & & SP & & \\
\hline & Orchis pallens L. & 8 & G & VU & SP & & \\
\hline & Orchis simia Lam. & 1 & G & VU & SP & & \\
\hline & Platanthera bifolia (L.) Rich. & 9 & G & VU & SP & & \\
\hline Orobanchaceae & Orobanche crenata Forssk. & 3 & $\mathrm{~T}$ & & & & \\
\hline Oxalidaceae & Oxalis acetosella $\mathrm{L}$. & 9 & $\mathrm{H}$ & & & & \\
\hline Papaveraceae & Chelidonium majus L. & 11 & $\mathrm{H}$ & & & & \\
\hline \multirow{2}{*}{ Plantaginaceae } & Plantago lanceolata $\mathrm{L}$. & 9 & $\mathrm{H}$ & & & & \\
\hline & Plantago major L. ssp. intermedia (Gilib.) Lange & 9 & $\mathrm{H}$ & & & & \\
\hline \multirow{15}{*}{ Poaceae } & Alopecurus pratensis $\mathrm{L}$. & 9 & $\mathrm{H}$ & & & & \\
\hline & Anthoxanthum odoratum L. & 9 & $\mathrm{H}$ & & & & \\
\hline & Avena sterilis L. & 5 & $\mathrm{H}$ & & & & \\
\hline & Avenula pubescens (Dumort.) Dumort. & 9 & $\mathrm{H}$ & & & & \\
\hline & Brachypodium sylvaticum (Huds.) P. Beauv. & 9 & $\mathrm{H}$ & & & & \\
\hline & Briza media $\mathrm{L}$. & 9 & $\mathrm{H}$ & & & & \\
\hline & Cynosurus cristatus L. & 11 & $\mathrm{H}$ & & & & \\
\hline & Dactylis glomerata L. & 9 & $\mathrm{H}$ & & & & \\
\hline & Festuca pratensis Huds. & 9 & $\mathrm{H}$ & & & & \\
\hline & Holcus lanatus L. & 9 & $\mathrm{H}$ & & & & \\
\hline & Hordeum murinum L. ssp. murinum & 11 & $\mathrm{~T}$ & LC & & & \\
\hline & Lolium perenne L. & 8 & $\mathrm{H}$ & & & & \\
\hline & Melica uniflora Retz. & 7 & G & & & & \\
\hline & Poа аппиа $\mathrm{L}$. & 9 & $\mathrm{~T}$ & LC & & & \\
\hline & Poa pratensis L. & 10 & $\mathrm{H}$ & & & & \\
\hline
\end{tabular}




\begin{tabular}{|c|c|c|c|c|c|c|c|}
\hline Family & Taxa & 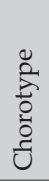 & 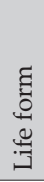 & 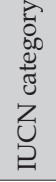 & 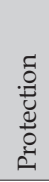 & 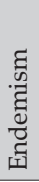 & 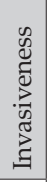 \\
\hline \multirow{2}{*}{ Poaceae } & Setaria viridis (L.) P. Beauv. & 9 & $\mathrm{~T}$ & & & & \\
\hline & Trisetum flavescens (L.) P. Beauv. & 10 & $\mathrm{H}$ & & & & \\
\hline Polygalaceae & Polygala comosa Schkuhr & 9 & $\mathrm{H}$ & & & & \\
\hline \multirow{4}{*}{ Polygonaceae } & Polygonum aviculare L. & 11 & $\mathrm{~T}$ & & & & \\
\hline & Rumex acetosa $\mathrm{L}$. & 9 & $\mathrm{H}$ & & & & \\
\hline & Rumex crispus L. & 9 & $\mathrm{H}$ & & & & \\
\hline & Rumex obtusifolius L. & 9 & $\mathrm{H}$ & & & & \\
\hline \multirow{4}{*}{ Primulaceae } & Anagallis arvensis L. & 11 & $\mathrm{~T}$ & & & & \\
\hline & Cyclamen purpurascens Mill. & 2 & G & NT & & & \\
\hline & Lysimachia nummularia L. & 8 & $\mathrm{H}$ & & & & \\
\hline & Primula vulgaris Huds. & 10 & $\mathrm{H}$ & & & & \\
\hline \multirow{10}{*}{ Ranunculaceae } & Aconitum lycoctonum L. ssp. vulparia (Rchb.) Nyman & 9 & $\mathrm{H}$ & & & & \\
\hline & Actaea spicata L. & 9 & $\mathrm{H}$ & & & & \\
\hline & Anemone nemorosa L. & 7 & G & & & & \\
\hline & Clematis vitalba $\mathrm{L}$. & 7 & $\mathrm{P}$ & & & & \\
\hline & Helleborus atrorubens Waldst. et Kit. & 2 & G & LC & SP & $\mathrm{E}$ & \\
\hline & Hepatica nobilis Schreber & 7 & $\mathrm{H}$ & & & & \\
\hline & Isopyrum thalictroides L. & 9 & G & & & & \\
\hline & Ranunculus acris L. & 9 & $\mathrm{H}$ & & & & \\
\hline & Ranunculus ficaria L. & 7 & $\mathrm{H}$ & & & & \\
\hline & Ranunculus lanuginosus L. & 7 & $\mathrm{H}$ & & & & \\
\hline Rhamnaceae & Rhamnus cathartica L. & 9 & $\mathrm{P}$ & & & & \\
\hline \multirow{20}{*}{ Rosaceae } & Agrimonia eupatoria L. & 10 & $\mathrm{H}$ & & & & \\
\hline & Aruncus dioicus (Walter) Fernald & 11 & $\mathrm{H}$ & & & & \\
\hline & Crataegus monogyna Jacq. & 9 & $\mathrm{P}$ & & & & \\
\hline & Filipendula vulgaris Moench & 9 & $\mathrm{H}$ & & & & \\
\hline & Fragaria moschata Duchesne & 7 & $\mathrm{H}$ & & & & \\
\hline & Fragaria vesca L. & 11 & $\mathrm{H}$ & & & & \\
\hline & Geum urbanum L. & 9 & $\mathrm{H}$ & & & & \\
\hline & Malus domestica Borkh. & 12 & $\mathrm{P}$ & & & & \\
\hline & Potentilla inclinata Vill. & 9 & $\mathrm{H}$ & & & & \\
\hline & Potentilla micrantha Ramond ex DC. & 3 & $\mathrm{H}$ & & & & \\
\hline & Potentilla reptans $\mathrm{L}$. & 11 & $\mathrm{H}$ & & & & \\
\hline & Prunus avium L. & 9 & $\mathrm{P}$ & & & & \\
\hline & Prunus domestica L. & 12 & $\mathrm{P}$ & & & & \\
\hline & Prunus spinosa $\mathrm{L}$. & 9 & $\mathrm{P}$ & & & & \\
\hline & Pyrus communis L. & 12 & $\mathrm{P}$ & & & & \\
\hline & Rosa canina L. & 9 & $\mathrm{P}$ & & & & \\
\hline & Rubus caesius L. & 9 & $\mathrm{P}$ & & & & \\
\hline & Sanguisorba minor Scop. & 9 & $\mathrm{H}$ & & & & \\
\hline & Sorbus aria (L.) Crantz & 11 & $\mathrm{P}$ & & & & \\
\hline & Sorbus aucuparia L. & 9 & $\mathrm{P}$ & & & & \\
\hline \multirow{4}{*}{ Rubiaceae } & Cruciata glabra (L.) Ehrend. & 3 & $\mathrm{H}$ & & & & \\
\hline & Galium aparine $\mathrm{L}$. & 9 & $\mathrm{~T}$ & & & & \\
\hline & Galium lucidum All. & 3 & $\mathrm{H}$ & & & & \\
\hline & Galium mollugo L. & 9 & $\mathrm{H}$ & & & & \\
\hline
\end{tabular}




\begin{tabular}{|c|c|c|c|c|c|c|c|}
\hline Family & Taxa & 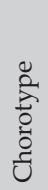 & 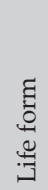 & $\begin{array}{l}2 \\
0 \\
0 \\
0 \\
0 \\
Z \\
Z \\
己\end{array}$ & 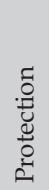 & 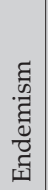 & 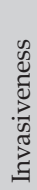 \\
\hline \multirow{3}{*}{ Rubiaceae } & Galium odoratum (L.) Scop. & 9 & G & & & & \\
\hline & Galium sylvaticum L. & 7 & G & & & & \\
\hline & Galium verum L. & 11 & $\mathrm{H}$ & & & & \\
\hline Salicaceae & Salix ${ }^{\times}$sepulcralis Simonk. & 12 & $\mathrm{P}$ & & & & \\
\hline \multirow{9}{*}{ Scrophulariaceae } & Lathraea squamaria $\mathrm{L}$. & 9 & G & & & & \\
\hline & Linaria vulgaris Mill. & 9 & G & & & & \\
\hline & Melampyrum sylvaticum L. & 8 & $\mathrm{~T}$ & & & & \\
\hline & Scrophularia nodosa L. & 10 & $\mathrm{H}$ & & & & \\
\hline & Veronica anagalloides Guss. & 3 & $\mathrm{~T}$ & & & & \\
\hline & Veronica chamaedrys L. & 9 & $\mathrm{Ch}$ & & & & \\
\hline & Veronica persica Poir. & 12 & $\mathrm{~T}$ & & & & I \\
\hline & Veronica serpyllifolia L. & 9 & $\mathrm{H}$ & & & & \\
\hline & Veronica urticifolia Jacq. & 7 & $\mathrm{Ch}$ & & & & \\
\hline Thymelaeaceae & Daphne mezereum L. & 9 & $\mathrm{P}$ & NT & & & \\
\hline Tiliaceae & Tilia platyphyllos Scop. & 7 & $\mathrm{P}$ & & & & \\
\hline Ulmaceae & Ulmus laevis Pall. & 7 & $\mathrm{P}$ & & & & \\
\hline Urticaceae & Urtica dioica $\mathrm{L}$. & 9 & $\mathrm{H}$ & & & & \\
\hline Violaceae & $\begin{array}{l}\text { Viola alba Besser } \\
\text { Viola reichenbachiana Jord. ex Boreau }\end{array}$ & $\begin{array}{l}3 \\
7\end{array}$ & $\begin{array}{l}\mathrm{H} \\
\mathrm{H}\end{array}$ & & & & \\
\hline Vitaceae & Vitis vinifera $\mathrm{L}$. & 11 & $\mathrm{P}$ & & & & \\
\hline
\end{tabular}

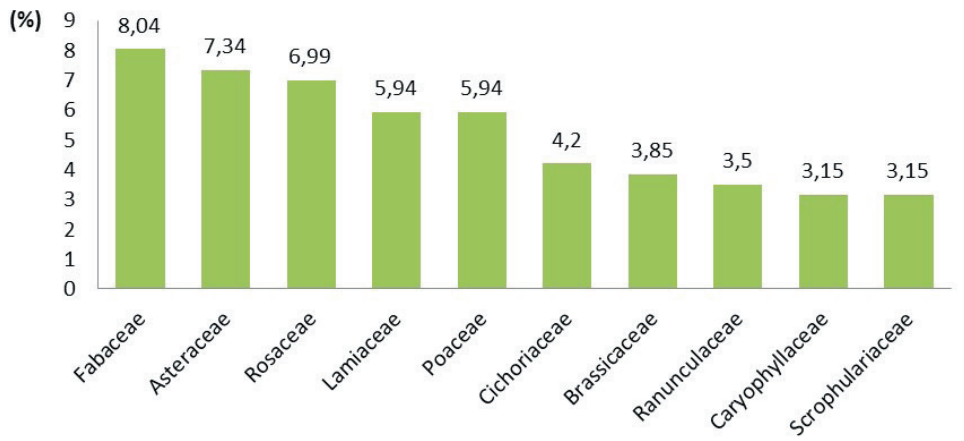

Fig. 2. The percentages of the most abundant families in the flora of trail "Okićnica".

The predominant life form is hemicryptophytes, which account for $52.96 \%$ of the taxa, reflecting the moderate climate of the wider area, followed by phanerophytes $(16.38 \%)$ and geophytes (15.68\%) (Fig. 3).

The chorological analyses placed the taxa within 12 floristic elements: Mediterranean, Illyrian-Balkan, South European, Atlantic, East European-Pontic, Southeast European, Central European, European, Eurasiatic, Circumholoarctic, cosmopolites and adventive and cultivated taxa. The dominance of Eurasiatic taxa (39.02\%), followed by Central European (13.94\%) and South European taxa (10.80\%) (Fig. 4) reflects the phytogeographical position of the researched area. Žumberačko Gorje Mts and Samobor- 


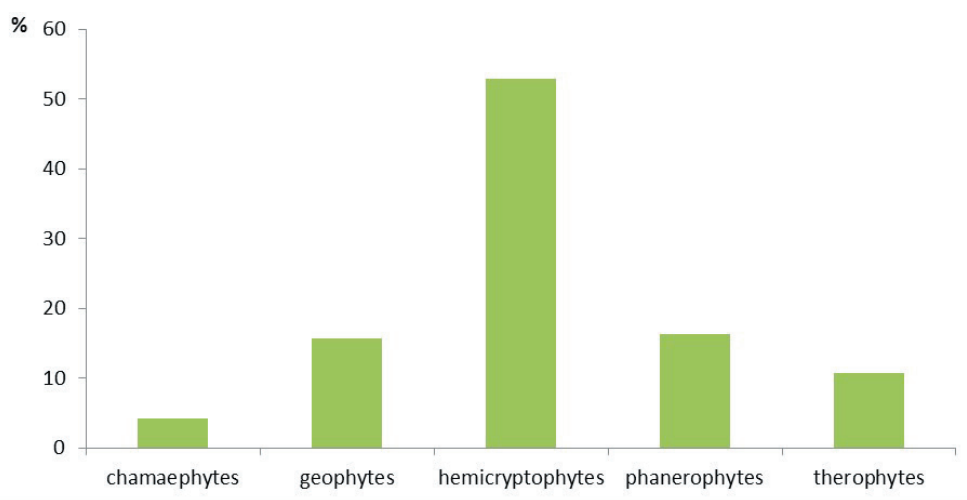

Fig. 3. Life form spectrum of the flora of trail "Okićnica".

sko gorje Mts are situated on the western border of the Pannonian plain, constituting a bridge between the Dinarides and Alps, resulting in miscellaneous plant taxa of diverse origins and recent distribution (Trinajstić, 1995). Trinajstić $(1992,1995)$ described a distinct Illyricoid chorotype on the boundary between the SE Alps and NW Dinarides, encompassing Tertiary relict flora, extinct in Central Europe during the Ice Ages, which survived in some refugia within mesophilic beech forests. The representatives of this flora are represented in the flora of the researched area by Aposeris foetida, Cardamine ennaeaphyllos, Cardamine trifolia, Cyclamen purpurascens, Euphorbia dulcis, Geranium phaeum, Hacquetia epipactis, Helleborus atrorubens, Knautia drymeia, Lamium orvala and Vicia oroboides.

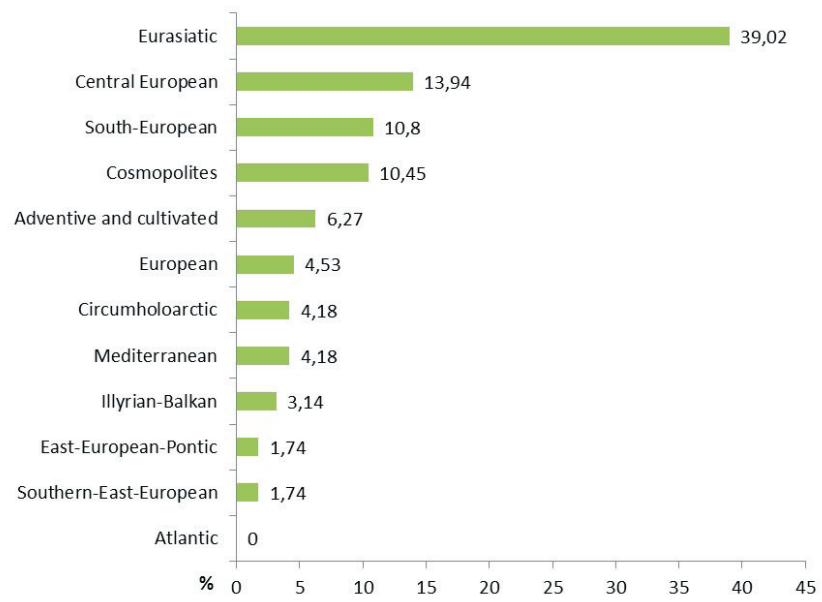

Fig. 4. The percentages of chorological types in the flora of trail "Okićnica”.

As for threatened taxa, in the flora of the trail six vulnerable (VU) (Dianthus giganteus subsp. croaticus, Ilex aquifolium, Lilium martagon, Orchis pallens, Orchis simia and Platanthera bifolia) and five near threatened (NT) taxa (Ruscus hypoglossum, Gentiana asclepiadea, Himantoglossum adriaticum, Cyclamen purpurascens and Daphne mezereum) 
were found. All of them are strictly protected by law, as are an additional three taxa (Helleborus atrorubens, Neottia nidus-avis and Orchis morio subsp. picta). Two of them (Dianthus giganteus subsp. croaticus and Helleborus atrorubens) are endemic species.

Along the trail, nine invasive taxa (3.14\%) were registered (Ambrosia artemisiifolia, Artemisia verlotiorum, Chamomilla suaveolens, Conyza canadensis, Erigeron annuus, Solidago gigantea, Lepidium virginicum, Robinia pseudoacacia and Veronica persica), six of them belonging to the family Asteraceae. None of these taxa show pronounced invasive capacity in the researched area, since the trail runs through predominantly forest vegetation, into which aliens rarely intrude and expand. Moreover, the trail has hitherto seldom been used by visitors and hikers, making the unintentional spread of alien species less likely.

\section{CONCLUSIONS}

Plant diversity along the Okićnica Trail has a high potential to be used for education in a natural environment. Special emphasis could be given to relict taxa of beech forests on the NW border of the Western Dinarides, as well as orchids as representatives of endangered and protected taxa. It would not be difficult to present the use of indigenous plants in local folk medicine and the traditional cuisine. Within the already existing promotion of the ethnological heritage of this particular region, such a presentation could be combined with advocacy for the preservation of the grasslands as local biodiversity hotspots.

\section{REFERENCES}

Alegro, A., Bogdanović, S., Rešetnik, I., Boršić, I., Cigić, P. \& Nikolić, T., 2013: Flora of the seminatural marshland Savica, part of the (sub)urban flora of the city of Zagreb (Croatia). Natura Croatica 22(1), 111-134.

Alegro, A., ŠEgota, V. \& PAPP, B., 2015: A contribution to the bryophyte flora of Croatia IV. Žumberačka gora Mts. Studia botanica hungarica 46(1), 5-24.

Anonymous, 2013: Pravilnik o strogo zaštićenim vrstama. Narodne novine 144/13.

Anonymous, 2016: Pravilnik o izmjenama i dopunama Pravilnika o strogo zaštićenim vrstama (Narodne novine 73/2016)

Budisavljević, A., Terlević, A., Mihelić, P., Špadina, B., Papković, D., Nikolić, T. \& Šegota V., 2017 : Vaskularna flora šume Dotrščina (Zagreb, Hrvatska). Glasnik Hrvatskog botaničkog društva 5(1), 4-19.

BuzjaK, S., KLETEČKI, N., Mitić, B. \& Vujnović, T., 2010: Flora ulaznih dijelova nekih jama i spilja Žumberka (Hrvatska). Natura Croatica: Periodicum Musei Historiae Naturalis Croatici, 19(1), 165-177.

BuzJaK, N., 2011: Geomorfološka obilježja. In: Feletar, D. (ed.): Samobor - zemljopisno-povijesna monografija, Knjiga 1. Meridijani, 45-49.

BuzJAK, N., BuzJaK, S. \& Orešıć, D., 2011: Florističke, mikroklimatske i geomorfološke značajke ponikve Japage na Žumberku (Hrvatska). Šumarski list, 135(3), 291-305.

Delforge, P., 1995: Orchis of Britain and Europe. Harper Collins Publishers, London.

Doмac, R., 1994: Flora Hrvatske: priručnik za određivanje bilja. Školska knjiga, Zagreb.

EggenberG, S. \& MöHL, A, 2007: Flora Vegetativa. Ein Bestimmungsbuch für Pflanzen der Schweiz im blütenlosen Zustand. Haupt Ver., Bern-Stuttgart-Wien.

Ellenberg, H. \& C. Leuschner, 2010: Zeigerwerte der Pflanzen Mitteleuropas, Vegetation Mitteleuropas mit den Alpen, Chapter 27, UTB GmbH, Stuttgart.

Horvat, I., 1929: Rasprostranjenost i prošlost mediteranskih, ilirskih i pontskih elemenata u flori sjeverne Hrvatske i Slovenije. Acta Botanica Croatica 4, 1-34.

Horvat, I., 1962: Vegetacija planina zapadne Hrvatske. Prirodoslovna istraživanja JAZU 30, Zagreb.

Horvatić, S., 1954: Ilustrirani bilinar. Školska knjiga, Zagreb. 
Horvatić, S., 1963: Vegetacijska karta otoka Paga s općim pregledom vegetacijskih jedinica hrvatskog primorja. Prirodoslovna istraživanja serija Acta Biologica 4(33), 5-181.

Horvatić, S., Ilijanić, Lj. \& Marković-Gospodarić, Lj., 1967-1968: Biljni pokrov okoline Senja. Senjski zbornik 3, 298-323.

Javorka, S. \& CsApody, V., 1991: Iconographiae florae partis Austro-orientalis Europae centralis. Akademiaa Kiado, Budapest.

Landolt, E., Bäumler, B., Erhardt, A., Hegg, O., Klötzli, F., Lämmler, W., Nobis, M., Rudmann-Maurer, K., Schweingruber, F. H., Theurillat, J-P., Urmi, E., Vust, M. \& Wohlgemuth, Th., 2010: Flora Indicativa. Ökologische Zeigerwerte und biologische Kennzeichen zur Flora der Schweiz und der Alpen. Editions des Conservatoire et Jardin botaniquesde la Ville de Geneve and Haupt Verlag, BernStuttgart-Wien.

Niкolıć, T. \& Topıć, J. (eds.), 2005: Crvena knjiga vaskularne flore Hrvatske. Ministarstvo kulture, Državni zavod za zaštitu prirode, Zagreb.

Nikolić, T. (ed.), 2020: Flora Croatica Database. On-Line (http://hirc.botanic.hr/fcd). Division of Botany, Faculty of Science, University of Zagreb, accessed on 1 January 2020.

Nikolić, T., Milović, M., Bogdanović, S. \& Jasprica, N., 2015: Endemi u hrvatskoj flori. Alfa d.d., Zagreb.

Nikolić, T., Mitić B. \& Boršić, I., 2014: Flora Hrvatske-invazivne biljke. Alfa d.d., Zagreb.

Penzar, B. \& Penzar, I., 1982: Prikaz godišnjeg hoda oborina u Hrvatskoj pomoću Köppenove sheme. Radovi (Šumarski institut) 17-18, 3-9.

Raunkiaer, C., 1934: The life forms of plants and statistical plant geography. The Clarendon Press, Oxford.

REŠETNIK, I. \& ŠEGOTA, V., 2020: Virtual herbarium ZA \& ZAHO. On-line (http://herbariumcroaticum.biol. pmf.hr). Division of Botany, Faculty of Science, University of Zagreb, accessed on 1 January 2020.

Rothmaler, W., 2000: Exkursionsflora von Deutschland. Spektrum Akademischer Verlag, Heidelberg-Berlin.

Šoštarić, R., Lucić, A., Caput, K., ĆAleta, M., Janev Hutinec, B., Lajtner, J. \& Vilenica, M., 2011: Inventorisation of flora and fauna of waterbodies on the territory of "Žumberak - Samoborsko gorje“ Nature park. Stručna studija, Prirodoslovno-matematički fakultet, Zagreb.

ŠošTarić, R., SEdlar, Z. \& MAReković, S., 2012: Ugroženi bazofilni cret uz potok Jarak (Park prirode Žumberak-Samoborsko gorje, Hrvatska). Natura Croatica: Periodicum Musei Historiae Naturalis Croatici, 21(2), 335-348.

Šoštarić, R., SEDlar, Z. \& MAReković, S., 2013: Flora i vegetacija Sopotskog slapa i gornjeg toka Kupčine (Park prirode Žumberak-Samoborsko gorje) s prijedlogom mjera zaštite. Glasnik Hrvatskog botaničkog društva, 1(2), 4-17.

ŠugAR, I., 1972: Biljni svijet Samoborskog Gorja, Doktorska disertacija, Prirodoslovno-matematički fakultet, Sveučilište u Zagrebu, Zagreb.

Trinajstić, I., 1992: Prilog fitogeografskom razgraničenju ilirskog flornog elementa. Acta Botanica Croatica, 51(1), 135-141.

TRINAJSTIĆ, I., 1995: Samoborsko gorje - refugijalno područje različitih flornih elemenata između Alpa i Dinarida. Acta Botanica Croatica, 54(1), 47-62.

VRbek, M. \& Fiedler, S., 1998: The distribution, degree of threat to and conservation of the orchids of Žumberak (Croatia). Natura Croatica 7(4), 291-305.

VRbeк, M., 2005: Flora i nešumska vegetacija Žumberka. Doktorska disertacija, Prirodoslovno-matematički fakultet Sveučilišta u Zagrebu.

Vukelić, J., 2012: Šumska vegetacija Hrvatske. Šumarski fakultet, Sveučilište u Zagrebu \& Državni zavod za zaštitu prirode, Zagreb, 403 pp.

Vuković, N., Boršić, I., Župan, D., Alegro, A. \& Nikolić, T., 2013: Vascular flora of Jarun (Zagreb, Croatia). Natura Croatica 22(2), 275-294. 
